\section{Fractional Fourier Series Expansion for Finite Signals and Dual Extension to Discrete-Time Fractional Fourier Transform}

Soo-Chang Pei, Min-Hung Yeh, and Tzyy-Liang Luo

\begin{abstract}
Conventional Fourier analysis has many schemes for different types of signals. They are Fourier transform (FT), Fourier series (FS), discrete-time Fourier transform (DTFT), and discrete Fourier transform (DFT). The goal of this correspondence is to develop two absent schemes of fractional Fourier analysis methods. The proposed methods are fractional Fourier series (FRFS) and discrete-time fractional Fourier transform (DTFRFT), and they are the generalizations of Fourier series (FS) and discrete-time Fourier transform (DTFT), respectively.
\end{abstract}

Index Terms-Discrete-time fractional Fourier transform, fractional Fourier series.

\section{INTRODUCTION}

The conventional Fourier analysis can obtain the frequency components of a signal [1], [2], and the fractional Fourier analysis can reveal the mixed time and frequency components of signals [3]. Until now, fractional Fourier analysis has only two schemes to deal with different types of signals. The existing schemes are fractional Fourier transform (FRFT) and discrete fractional Fourier transform (DFRFT) [4], [5]. They can be used for the continuous and discrete signals, and their results are continuous and discrete, respectively. Until now, several methods for computing the DFRFT of signal have been proposed [4], [5]. The method developed in [5] cannot provide a result to match its continuous corresponding case. A rigorous discussion for the mismatches of [5] has been presented in [6]. In this correspondence, we will use the DFRFT in [4] for the further discussions.

\section{REVIEW OF THE Fractional Fourier Transform}

The transform kernel of the continuous fractional Fourier transform (FRFT) is defined as follows [3]:

$$
K_{\alpha}(t, u)= \begin{cases}\sqrt{\frac{1-j \cot \alpha}{2 \pi}} e^{j\left(\left(t^{2}+u^{2}\right) / 2\right) \cot \alpha-j t u \csc \alpha} \\ \delta(t-u), \quad \text { if } \alpha \text { is not a multiple of } \pi \\ \delta(t+u), \quad \text { if } \alpha+\pi \text { is a multiple of } 2 \pi\end{cases}
$$

where $\alpha$ indicates the rotation angle of the transformed signal for FRFT. The forward and inverse FRFT are defined as

$$
\begin{aligned}
\mathcal{X}_{\alpha}(u) & =\int_{-\infty}^{\infty} x(t) K_{\alpha}(t, u) d t \\
x(t) & =\int_{-\infty}^{\infty} \mathcal{X}_{\alpha}(u) K_{-\alpha}(t, u) d u .
\end{aligned}
$$

The properties of FRFT have been summarized in [3]. The domains $0<\alpha<(\pi / 2)$ are called fractional Fourier domains in [7], and this definition is also adopted in this paper.

Manuscript received January 27, 1998; revised March 10, 1999. The associate editor coordinating the review of this paper and approving it for publication was Dr. Konstantinos Konstantinides.

S.-C. Pei and T.-L. Luo are with the Department of Electrical Engineering, National Taiwan University, Taipei, Taiwan, R.O.C.

M.-H. Yeh is with the Department of Computer Information Science, Tamsui Oxford University College, Tamsui, Taipei, Taiwan, R.O.C.

Publisher Item Identifier S 1053-587X(99)07664-3.

\section{Fractional Fourier Series for Finite Signals}

The frequency spectrum of a periodic signal consists of a sequence of impulse. This leads to the Fourier series expansion of signal. The Fourier series expansion is defined as [1]

$$
x(t)=\sum_{n=-\infty}^{\infty} C_{n} e^{j n t \omega_{0}}
$$

where $x(t)$ is a periodic signal with period $T$ or a finite signal with duration $T \cdots \omega_{0}=(2 \pi / T)$. The FS expansion coefficients can be computed as

$$
C_{n}=\frac{1}{T} \int_{T} x(t) e^{-j n t \omega_{0}} d t
$$

where $n=-\infty, \cdots,-1,0,1, \cdots, \infty$. The FS expansion can be used for two classes of continuous signals [1]. One can represent an aperiodic signal $x(t)$ on a finite interval, say, $[0, T]$. In this case, the Fourier series converge to the periodic extension of $x(t)$, outside of $[0, T]$, that is, to $x(t+n T), n= \pm 1, \pm 2, \cdots$. We can also use the FS to represent a periodic signal with any period.

In developing fractional Fourier series (FRFS), we first find the orthogonal basis. In the conventional FS case, a basis function-sinusoids - has an impulse output in the Fourier domain. We can use the same notation to find the FRFS basis functions. Because an impulse function $\delta\left(t-n t_{0}\right)$ in the fractional Fourier domain with parameter $\alpha$ whose original time domain representation is

$$
\begin{aligned}
& \text { FRFT }_{-\alpha}\left[\delta\left(t-n t_{0}\right)\right] \\
& \quad=\sqrt{\frac{1+j \cot \alpha}{2 \pi}} e^{-j\left(\left(t^{2}+\left(n t_{0}\right)^{2}\right) / 2\right) \cot \alpha+j\left(n t_{0}\right) t \csc \alpha}
\end{aligned}
$$

the definition of the basis signals of FRFS that we used is listed as

$$
\phi_{\alpha, n}(t)=\sqrt{\frac{1+j \cot \alpha}{2 \pi}} e^{-j\left(\left(t^{2}+\left(n t_{0}\right)^{2}\right) / 2\right) \cot \alpha+j\left(n t_{0}\right) t \csc \alpha}
$$

where $t_{0}$ is called the central frequency in the fractional Fourier domains. The basis of FRFS is a chirp signal that is a not periodic function, and the summation of aperiodic functions cannot attain a periodic one; therefore, the FRFS is only for finite signals. After the basis signals of FRFS are determined, we can compute the integration for any two basis signals along the whole interval $[-(T / 2),(T / 2)]$ to check the orthogonal property in (8) and (9), shown at the bottom of the next page, where $\tilde{t}=t \csc \alpha$. If we want $\phi_{\alpha, n}$ to satisfy the orthogonal condition, the right part of the (9) in the case $m \neq n$ should equal zero. This condition can be satisfied only when $t_{0}=2 \pi(\sin \alpha / T)$. Moreover, we can divide each $\phi_{\alpha, n}$ by the value $\sqrt{T \csc \alpha /(2 \pi)}$ in order to obtain an orthonormal basis $\left(\tilde{\phi}_{\alpha, n}\right)$ of FRFS.

$$
\begin{aligned}
\tilde{\phi}_{\alpha, n}(t)= & \frac{\phi_{\alpha, n}(t)}{\sqrt{\frac{T \csc \alpha}{2 \pi}}}=\sqrt{\frac{\sin \alpha+j \cos \alpha}{T}} \\
& \cdot e^{-j\left(\left(t^{2}+(n(2 \pi / T) \sin \alpha)^{2}\right) / 2\right) \cot \alpha+j n t(2 \pi / T)}
\end{aligned}
$$

$\underset{\sim}{\text { where }} n=-\infty, \cdots,-1,0,1, \cdots, \infty$. Thus, $\left\{\cdots, \tilde{\phi}_{\alpha,-1}, \tilde{\phi}_{\alpha, 0}\right.$, $\left.\tilde{\phi}_{\alpha, 1}, \cdots\right\}$ construct an orthonormal basis. The instantaneous frequency of basis signal can be obtained by computing the derivative of its phase. Therefore, it is equal to

$$
\omega_{\alpha, n}(t)=-t \cot \alpha+n \frac{2 \pi}{T} .
$$


TABLE I

PROPERTIES OF FRFS

\begin{tabular}{|c|c|}
\hline Reversal & $F R F S[x(-t)]=C_{\alpha,(-n)}$ \\
\hline Conjugation & $F R F S\left[x^{*}(t)\right]=j C_{(-\alpha), n}^{*}$ \\
\hline Shift & $\begin{array}{l}\tau=k \frac{2 \pi \sin \alpha}{T}, k \text { is an integer. } t_{0}=2 \pi \sin \alpha / T \\
F R F S[x(t-\tau)]=C_{\alpha,(n-k)} e^{j \frac{\tau^{2}}{2} \sin \alpha \cos \alpha-j n t_{0} \tau \sin \alpha}\end{array}$ \\
\hline Modulation & $\begin{array}{l}F R F S\left[x(t) e^{j m t_{0} t}\right]=e^{-\frac{\left(m x_{0}\right)^{2}}{2}} \sin \alpha \cos \alpha-j m n t_{0}^{2} \cos \alpha{ }_{\alpha,(n-m \sin \alpha)} \\
\text { where } t_{0}=2 \pi \sin \alpha / T \text {, and }(n-m \sin \alpha) \text { is an integer. }\end{array}$ \\
\hline Scaling & $\begin{array}{l}\text { FRFS }[x(c t)]=\sqrt{c} \sqrt{\frac{\sin \alpha}{\sin \beta}} \sqrt{\frac{1-j \cot \alpha}{c^{2}-j \cot \alpha}} e^{\frac{j\left(n t_{0}\right)^{2} \cot \beta}{2}\left(c^{4}-\frac{\sin ^{2} \beta}{\sin ^{2} \alpha}\right)} C_{\beta, n} \\
\text { where } \beta=\tan ^{-1}\left(c^{2} \tan \alpha\right) \cdot t_{0}=2 \pi \sin \alpha / T . \\
\text { The central frequency of FRFS }[\mathrm{x}(c \mathrm{ct})] \text { equals } t_{0}^{\prime}=2 \pi \sin \beta / T\end{array}$ \\
\hline Parseval & $\alpha \neq m \pi$ and $m$ are integers, $\sum_{n=-\infty}^{\infty}\left|C_{\alpha, n}\right|^{2}=\int_{T}|x(t)|^{2} d t$ \\
\hline
\end{tabular}

From (11), it can be observed that every basis signal is a chirp signal with chirp rate $(-\cot \alpha)$. The interpretation of the FRFS expansion for a finite signal is that the latter is decomposed in terms of its chirp harmonics. The well-known Fourier series (FS) is just a special case of FRFS for the case $\alpha=(\pi / 2)$, and the basis signals of FS are sinusoids harmonics that are horizontal lines in the time-frequency plane. Thus, the FRFS expansion of the finite signal $x(t)$ can be written as

$$
\begin{aligned}
x(t)= & \sum_{n=-\infty}^{\infty} C_{\alpha, n} \tilde{\phi}_{\alpha, n}(t) \\
= & \sum_{n=-\infty}^{\infty} C_{\alpha, n} \sqrt{\frac{\sin \alpha+j \cos \alpha}{T}} \\
& \cdot e^{-j\left(\left(t^{2}+(n(2 \pi / T) \sin \alpha)^{2}\right) / 2\right) \cot \alpha+j n t(2 \pi / T)} \\
& \quad t \in\left[-\frac{T}{2}, \frac{T}{2}\right]
\end{aligned}
$$

where $C_{\alpha, n}$ are called FRFS expansion coefficients with the parameter $\alpha$. The FRFS expansion coefficients are computed by the inner product of the signal and chirp basis signals.

$$
\begin{aligned}
C_{\alpha, n}= & \int_{-(T / 2)}^{T / 2} x(t) \tilde{\phi}_{\alpha, n}^{*}(t) d t \\
= & \sqrt{\frac{\sin \alpha-j \cos \alpha}{T}} \int_{-(T / 2)}^{T / 2} x(t) \\
& \cdot e^{j\left(\left(t^{2}+(n(2 \pi / T) \sin \alpha)^{2}\right) / 2\right) \cot \alpha-j n t(2 \pi / T)} d t .
\end{aligned}
$$

The FRFS expansion can be used to represent an aperiodic signal $x(t)$ on a finite interval, say, $[-(T / 2),(T / 2)]$.

The properties of FRFS are shown in Table I. From Table I, it follows that the FRFS has similar properties as those of FRFT, but the shift and modulation properties of FS and FRFS are summarized in Table III with some constraints.

It has been proved in Appendix A that the expansion coefficients of FRFS can be obtained from the sampled values of FRFT of $x(t)$

$$
C_{\alpha, n}=\sqrt{\frac{2 \pi \sin \alpha}{T}} \mathcal{X}_{\alpha}\left(n \frac{2 \pi}{T} \sin \alpha\right)
$$

where $C_{\alpha, n}$ are FRFS expansion coefficients of the signal $x(t) . \mathcal{X}_{\alpha}(\cdot)$ is the FRFT of the signal $x(t)$. From (14), we know that the sampling interval depends on $\alpha$ as well as $T$. When the computing interval of FRFS approaches infinity, the sampling spacing of FRFS coefficients will also approach zero. Therefore, the FRFS will converge to the FRFT while the computing interval $T$ approaches infinity.

Example 1: In this example, we compute the FRFS coefficients for the chirp signal $\left(x(t)=e^{-j t^{2}}\right.$ for $|t| \leq \pi, x(t)=0$ otherwise). From the previous results in FRFT, we know that the FRFT output of the chirp signal $e^{-j c t^{2}}$ will become an ideal impulse for the specific angle $\tan ^{-1}(1 / 2 c)$ [3], [10], [11]. In the FRFS expansion case, the finite chirp signal $e^{-j c t^{2}}$ can have only one nonzero impulse at $n=0$ for the case $\alpha=\tan ^{-1}(1 / 2 c)$ because only one basis signal is enough to synthesize the chirp signal. Fig. 1 shows the finite chirp signal $x(t)$ and its FRFS expansions. In this correspondence, the real parts of signal and transforms are plotted by solid lines, and the imaginary parts are plotted by dashed lines. When the angle $\alpha=\tan ^{-1}(0.5) \approx 0.4636$, the FRFS coefficients has only one nonzero impulse with value $C_{\alpha, 0}=2.132-1.318 j$. Moreover, we compute the FRFS with a closer angle of $\tan ^{-1}(0.5)$. While the angle $\alpha=(3 \pi / 20) \approx 0.4712$, the FRFS expansion has only three significant values $(n=-1,0,1) . C_{\alpha, 0}=2.05-1.436 j$, $C_{\alpha, 1}=C_{\alpha,-1}=0.03817+0.0855 j$. Fig. 2 shows the reconstructed signal that is synthesized by three major FRFS components $(n=$ $-1,0,1)$. From the plot in Fig. 2, we know that only three FRFS components can approximate the original chirp signal very well. The reconstruction error in this example is computed as

$$
r(t)=x(t)-\left(C_{\alpha, 0} \tilde{\phi}_{\alpha, 0}(t)+C_{\alpha, 1} \tilde{\phi}_{\alpha, 1}(t)+C_{\alpha,-1} \tilde{\phi}_{\alpha,-1}(t)\right)
$$

and the ratio of power of reconstruction error and signal is equal to

$$
\frac{\int_{T}|r(t)|^{2} d t}{\int_{T}|x(t)|^{2}}=\frac{0.0014525}{6.2832}=0.02312 \%
$$

\section{Discrete-Time Fractional Fourier Transform}

The well-known discrete-time Fourier transform (DTFT) provides a method for computing the frequency components of a discrete signal. The definition of DTFT in [2] are represented in frequency normalization notation, and they are periodical function with period $2 \pi$. Without frequency normalization, the transform result of DTFT should be a periodic function with period $2 \pi / T_{s}$, where $T_{s}$ is the sampling interval of the discrete signal. The definitions for the forward and inverse DTFT without frequency normalization should be changed into the following formulas.

$$
\begin{aligned}
\mathcal{X}(\nu) & =\sqrt{\frac{T_{s}}{2 \pi}} \sum_{n=-\infty}^{\infty} x[n] e^{-j n \nu T_{s}} \\
x[n] & =\sqrt{\frac{T_{s}}{2 \pi}} \int_{-\left(\pi / T_{s}\right)}^{\pi / T_{s}} \mathcal{X}(\nu) e^{j n \nu T_{s}} d \nu .
\end{aligned}
$$

The above definitions of forward and inverse DTFT will be used in this correspondence. In this section, we will develop the

$$
\begin{aligned}
& \int_{-(T / 2)}^{T / 2} \phi_{\alpha, m}(t) \phi_{\alpha, n}^{*}(t) d t \\
&= \frac{1}{2 \pi} e^{j\left(\left(\left(n t_{0}\right)^{2}-\left(m t_{0}\right)^{2}\right) / 2\right) \cot \alpha} \int_{-(T / 2) \csc \alpha}^{T / 2 \csc \alpha} e^{j(m-n) t_{0} \tilde{t}} d \tilde{t} \\
& \quad= \begin{cases}\frac{T \csc \alpha}{2 \pi}, & \text { when } m=n \\
\frac{1}{j 2 \pi(m-n) t_{0}} e^{j\left(\left(\left(n t_{0}\right)^{2}-\left(m t_{0}\right)^{2}\right) / 2\right) \cot \alpha} e^{-j(m-n) t_{0}(T / 2) \csc \alpha} \times\left\{e^{j(m-n) t_{0} T \csc \alpha}-1\right\}, & \text { when } m \neq n\end{cases}
\end{aligned}
$$


generalization of DTFT, which is discrete-time fractional Fourier transform (DTFRFT). The discrete signal $x[n]$ is sampled from the bandlimited signal $x(t)$ by sampling rate $T_{s}$. In the development of DTFRFT, the roles of time and frequency axis of FRFS are interchanged to define the DTFRFT. It means that the discrete samples in the time domain are treated as the FRFS coefficients in the frequency domain. The FRFS expansion for the spectrum $\mathcal{X}(-\nu)$ with the parameter $((\pi / 2)+\alpha)$ is performed. This concept is illustrated in Fig. 3. The choice of spectrum is $\mathcal{X}(-\nu)$ rather than $\mathcal{X}(\nu)$ because the FRFS expansion of $\mathcal{X}(-\nu)$ with positive angular parameter $((\pi / 2)+\alpha)$ can attach the DTFRFT solution. By (12), the spectrum of discrete sampled signal can be expanded as in (19), shown at the bottom of the page.

The FRFS expansion coefficients can be computed by (13) so that they can be obtained as

$$
\begin{aligned}
D_{\alpha}[n]= & C_{(\pi / 2)+\alpha, n} \\
= & \int_{-\left(\pi / T_{s}\right)}^{\pi / T_{s}} \mathcal{X}(-\nu) \sqrt{\frac{T_{s}}{2 \pi}(\cos \alpha+j \sin \alpha)} \\
& \times e^{-j\left(\left(\nu^{2}+n^{2} T_{s}^{2} \cos ^{2} \alpha\right) / 2\right) \tan \alpha} e^{-j n \nu T_{s}} d \nu \\
= & \frac{T_{s}}{2 \pi} \sqrt{\cos \alpha+j \sin \alpha} e^{-j\left(n^{2} T_{s}^{2} / 2\right) \sin \alpha \cos \alpha} \\
& \cdot \sum_{k=-\infty}^{\infty} x[k] \int_{-\left(\pi / T_{s}\right)}^{\pi / T_{s}} \\
& \cdot e^{-j\left(\nu^{2} / 2\right) \tan \alpha} e^{j \nu T_{s}(k-n)} d \nu \\
= & \frac{T_{s}}{2} \sqrt{\frac{1-j \cot \alpha}{2 \pi}} \sqrt{\cos \alpha} e^{-j\left(n^{2} T_{s}^{2} / 2\right) \sin \alpha \cos \alpha} \\
& \cdot \sum_{k=-\infty}^{\infty} x[k] \Gamma(n, k, \alpha)
\end{aligned}
$$

where $D_{\alpha}[n]$ is the DTFRFT of the discrete signal $x[k], \alpha \neq$ $m \pi+\pi / 2$ ( $m$ is an integer), and

$$
\begin{aligned}
\Gamma(n, k, \alpha)= & \left\{-\operatorname{erf}\left[j \frac{-\pi \tan \alpha+T_{s}^{2}(n-k)}{T_{s} \sqrt{2 j \tan \alpha}}\right]\right. \\
& \left.+\operatorname{erf}\left[j \frac{\pi \tan \alpha+T_{s}^{2}(n-k)}{T_{s} \sqrt{2 j \tan \alpha}}\right]\right\} \\
& \times e^{j\left(T_{s}^{2}(k-n)^{2} / 2\right) \cot \alpha}
\end{aligned}
$$

where the function $\operatorname{erf}(\cdot)$ is the well-known error function [12].

$$
\operatorname{erf}(t)=\frac{2}{\sqrt{\pi}} \int_{0}^{t} e^{-u^{2}} d u
$$

The method for computing the error function with complex argument is stated in [12], and the proof of (22) is shown in Appendix B.
When $(\alpha=0)$, the transform result $D_{\alpha}[n]$ will match the original signal $x[k]$. Although $(\alpha=m \pi+\pi / 2)$, the transform will be the conventional discrete-time Fourier transform, and it is a continuous result. Equation (20) provides a method for computing the DFRFT based on the frequency domain of the signal, and (22) provides a way to find the DTFRFT from the time domain of signal. The inverse DTFRFT transform is computed as

$$
\begin{aligned}
x[n]= & \frac{T_{s}}{2} \sqrt{\frac{1+j \cot \alpha}{2 \pi}} \sqrt{\cos \alpha} e^{j\left(n^{2} T_{s}^{2} / 2\right) \sin \alpha \cos \alpha} \\
& \times \sum_{k=-\infty}^{\infty} D_{\alpha}[k] \Gamma(n, k,-\alpha)
\end{aligned}
$$

where the auxiliary function $\Gamma(n, k, \alpha)$ has been defined in (23). From (25), it can be found that the original signal can be recovered from a DTFRFT with the angular parameter $(-\alpha)$.

Like in the FRFS case, the results of DTFRFT will approach FRFT while the sampling period $T_{s}$ approaches zero, and the result of DTFRFT can be obtained from the sampled values of FRFT.

$$
D_{\alpha}[n]=\sqrt{T_{s} \cos \alpha} \mathcal{X}_{\alpha}\left(n T_{s} \cos \alpha\right)
$$

where $\mathcal{X}_{\alpha}(\cdot)$ is the FRFT of the presampled signal $x(t)$ with the angular parameter $\alpha$. From (26), it can be observed that sampling spacing of DTFRFT will become smaller as the angular parameter approaches $(m \pi+(\pi / 2))$, where $m$ is an integer. If the parameter $\alpha$ equals $(m \pi+(\pi / 2))$, the sampling spacing of DTFRFT will become zero. This matches the well-known result that the output of DTFRFT is a continuous function.

The properties of DTFRFT listed in Table II are similar to those of FRFS, and only the angular parameter should be changed. Similar to the FRFS case, the modulation and shift properties of DTFRFT are also with some constraints, and the constraints are also summarized in Table III.

Table IV illustrates the signal types of different fractional Fourier schemes that have been developed to date. The last column in Table IV shows the types of conventional Fourier schemes. Among the four conventional schemes, the Fourier transform (FT) is the original method. The FS and DTFT have been discussed in the previous sections. The motivation of the DFT comes from the fact that the Fourier transform of a sampled and periodic signal will result in a sampled and periodic spectrum [1]. Therefore, the types of the original signal and transforms for DFT are both discrete and periodic. The type of DTFRFT adopted here is discrete and aperiodic in the fractional Fourier domains $(0<\alpha<\pi / 2)$. Someone may have a motivation of DTFRFT from the FRFT of $x(t)$

$$
\mathcal{F}_{\alpha}(x(t))=\mathcal{F}_{\alpha}\left(\sum_{n} x(n) \delta(t-n)\right) .
$$

$$
\begin{aligned}
\mathcal{X}(-\nu)= & \sum_{n=-\infty}^{\infty} C_{(\pi / 2)+\alpha, n} \sqrt{\frac{\sin \left(\frac{\pi}{2}+\alpha\right)+j \cos \left(\frac{\pi}{2}+\alpha\right)}{2 \pi / T_{s}}} \\
& \cdot e^{-j\left(\left(\nu^{2}+\left[n\left(2 \pi / 2 \pi / T_{s}\right) \sin ((\pi / 2)+\alpha)\right]^{2}\right) / 2\right) \cot ((\pi / 2)+\alpha)+j n \nu\left(2 \pi / 2 \pi / T_{s}\right)} \\
= & \sum_{n=-\infty}^{\infty} C_{(\pi / 2)+\alpha, n} \sqrt{\frac{\cos \alpha-j \sin \alpha}{2 \pi / T_{s}}} e^{j\left(\left(\nu^{2}+\left(n T_{s} \cos \alpha\right)^{2}\right) / 2\right) \tan \alpha+j n \nu T_{s}} .
\end{aligned}
$$




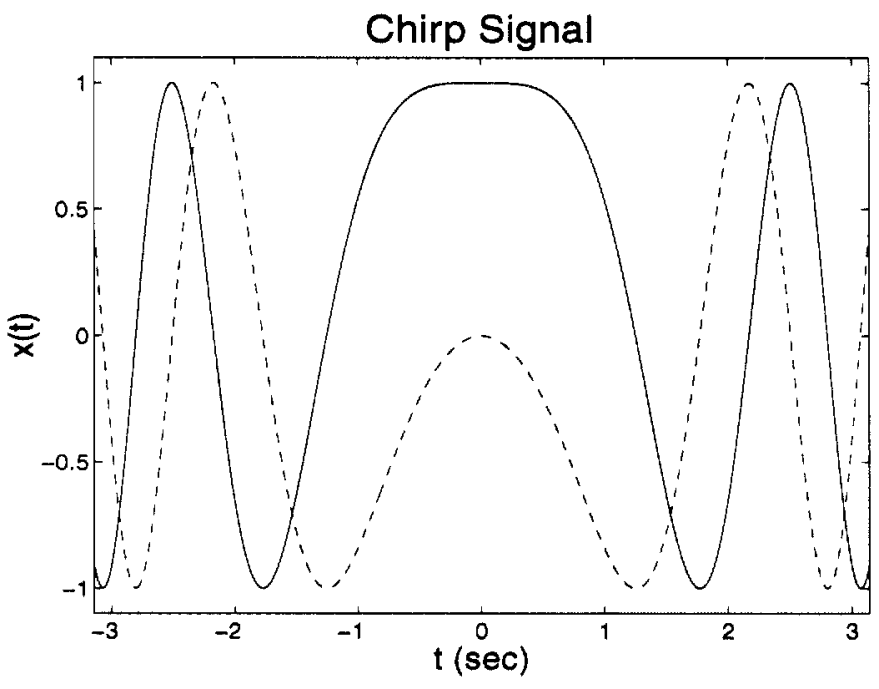

(a)

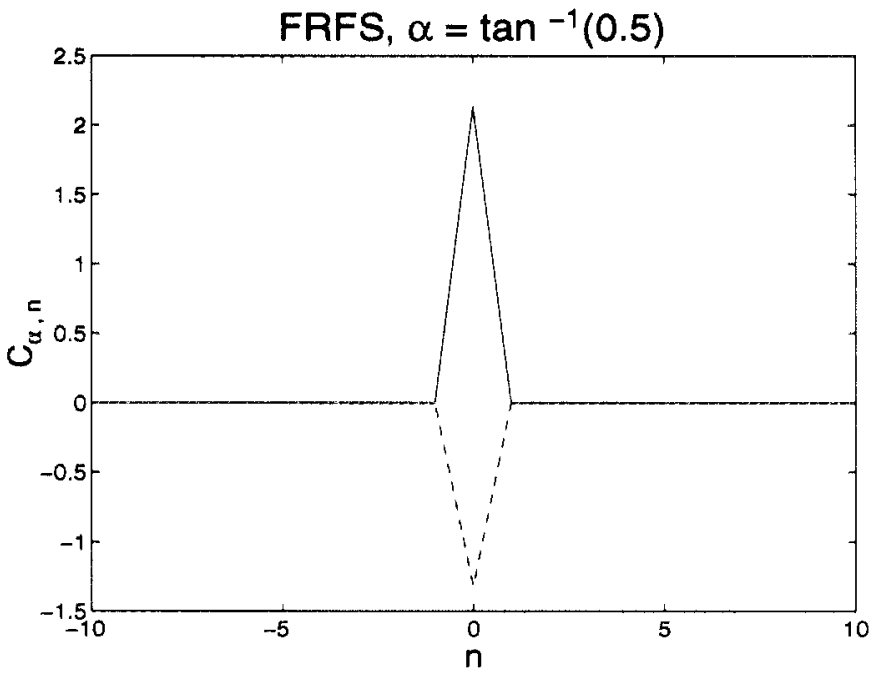

(b)

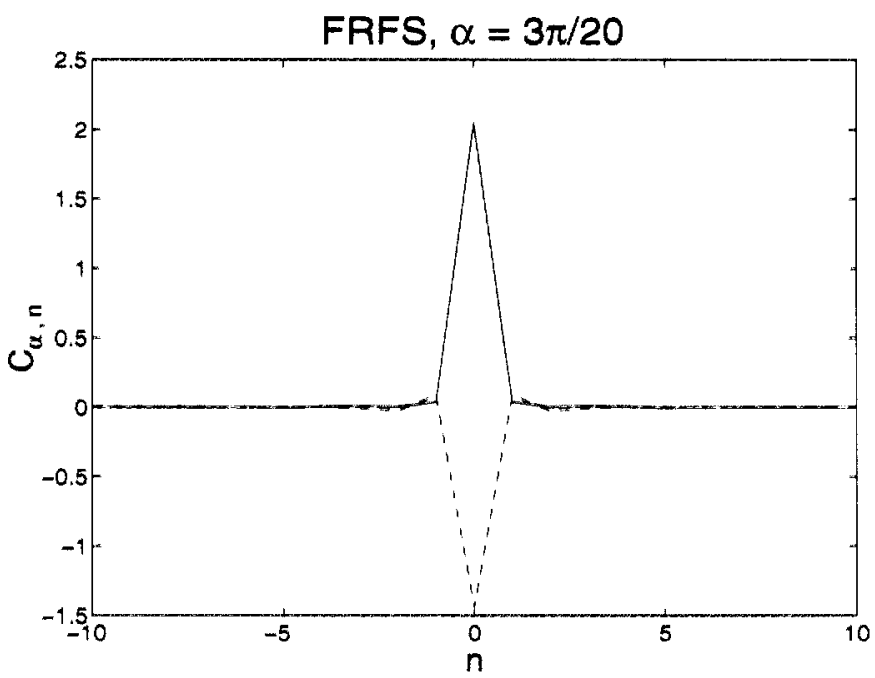

(c)

Fig. 1. (a) Finite chirp signal $\left(x(t)=e^{-j t^{2}}\right.$ for $|t| \leq \pi, x(t)=0$ otherwise). (b) FRFS expansion of $x(t)$ for $\alpha=\tan ^{-1}(0.5)$. (c) FRFS expansion of $x(t)$ for $\alpha=(3 \pi / 20)$.

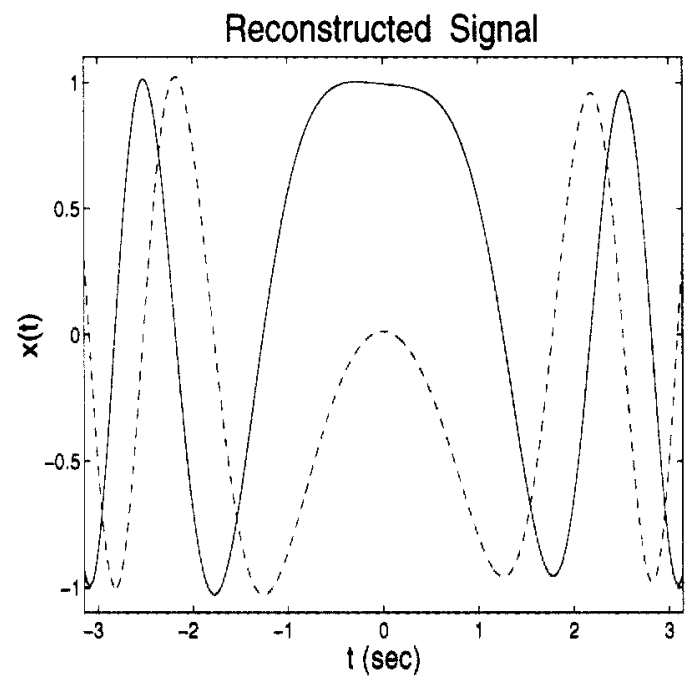

Fig. 2. Reconstructed signal synthesized by the three major FRFS components for the case $\alpha=(3 \pi / 20)$ in Example 1 .

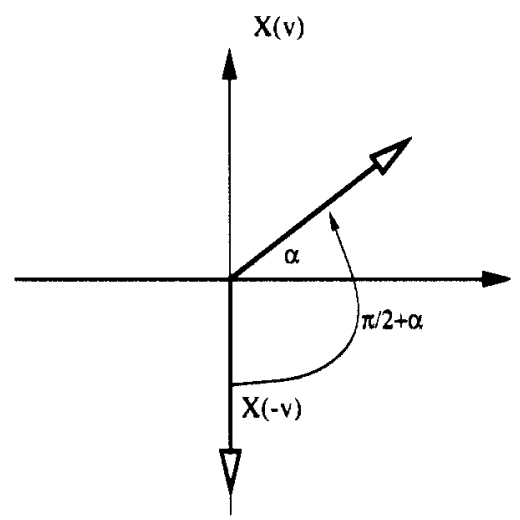

Fig. 3. Motivation of the DTFRFT derivation.

TABLE II

PROPERTIES OF DTFRFT

\begin{tabular}{|c|c|}
\hline Reversal & $D T F R F T(x[-n])=D_{\alpha}[-n]$ \\
\hline Conjugation & $D T F R F T\left(x^{*}[n]\right)=j D_{-\alpha, n}^{*}$ \\
\hline Shift & 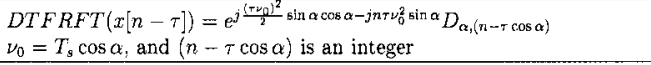 \\
\hline Modulation & $\begin{array}{l}T=k \times T_{3} \cot \alpha, \text { where the variable } k \text { is an integer. } \\
D T F R F T\left(x[n] e^{j n T_{s} \tau}\right)=D_{\alpha,(n-k)} e^{-j \frac{r^{2}}{2} \sin \alpha \cos \alpha+j n \nu_{0} \tau \cos \alpha}\end{array}$ \\
\hline Parseval & $(\alpha \neq m \pi+\pi / 2)$ and $m$ is an integer $\sum_{n=-\infty}^{\infty}|x[n]|^{2}=\sum_{n=-\infty}^{\infty} \mid D_{\alpha}[n]$ \\
\hline
\end{tabular}

TABLE III

Constraints of Shift and Modulation Properties of FRFS And DTFRFT

\begin{tabular}{c|c|c}
\hline & Shift Property & Modulation Property \\
\hline \hline & $x(t-\tau)$, & $x(t) e^{j n t}$, \\
FRFS & where $\tau=k \frac{2 \pi}{T} \tan \alpha, k$ is an integer & where $v=k \frac{2 \pi}{T}, k$ is an integer \\
\hline & $x(t-\tau)$, & $x(t) e^{j v t}$, \\
FS & $\tau$ is arbitrary & where $v=k \frac{2 \pi}{T}, k$ is an integer \\
\hline \hline & $x(n-\tau)$, & $x(n) e^{j n T_{n} v}$, \\
DTFRFT & $\tau$ and $(\tau \cos \alpha)$ are two integers & where $v=k T_{s}$ cot $\alpha_{1} k$ is an integer \\
\hline DTFT & $x(n-\tau), \tau$ is an integer & $x(n) e^{j n T_{s} v}, v$ is arbitrary \\
\hline
\end{tabular}


TABLE IV

Types of Signals Processed by the Different Fractional Schemes

\begin{tabular}{c|c|c|c}
\hline & $\begin{array}{c}\text { Time domain } \\
\alpha=0\end{array}$ & $\begin{array}{c}\text { Fractional Fourier domains } \\
0<\alpha<\pi / 2\end{array}$ & $\begin{array}{c}\text { Frequency domain } \\
\alpha=\pi / 2\end{array}$ \\
\hline FRFT & continuous & continuous & continuous (FT) \\
\hline FRFS & continuous, periodic & discrete, aperiodic & discrete, aperiodic (FS) \\
\hline DTFRFT & discrete, aperiodic & discrete, aperiodic & continuous, periodic (DTFT) \\
\hline DFRFT & discrete, periodic & discrete & discrete, periodic (DFT) \\
\hline
\end{tabular}

The above definition has the continuous output in fractional Fourier domain, but it cannot have good boundary conditions for the case $\alpha \approx 0$. Therefore, the type of DTFRFT adopted in this paper is discrete, aperiodic, and infinite in the fractional Fourier domain. Moreover, the spacing of DTFRFT in the fractional Fourier domain is $\left(T_{s} \cos \alpha\right)$. If $(\alpha=m \pi+\pi / 2)$, the spacing of DTFRFT will become zero, and the DTFRFT will be reduced to the conventional DTFT. About the FRFS case, the spacing is $(2 \pi \sin \alpha / T)$, and it will become zero while $(\alpha=m \pi)$. Thus, a continuous signal will be obtained. Moreover, the last row in Table IV presents the data type of the discrete fractional Fourier transform (DFRFT). Although this scheme is not discussed in detail in this correspondence, we still describe its signal types in Table IV.

\section{CONCLUSION}

From the above discussion, several conclusions can be made. First, FRFS is a generalization of FS, and it can reveal the mixed time and frequency components of signals. The FRFS expansion coefficients are the sampled values of FRFT. The envelope of FRFS coefficients will have a closer spacing as the computing interval increases. The FRFS will converge to FRFT when the computing interval $T$ approaches infinity. Second, the generalization of DTFT (DTFRFT) can be obtained through the dual extension of FRFS. The DFRFT can provide a method for computing the fractional Fourier transform for discrete signals. With the help of the algorithm of DTFRFT, the fractional Fourier analysis for discrete signals can be realized.

\section{APPENDIX A}

The FRFS coefficients of $x(t)$ can be computed as

$$
\begin{aligned}
C_{\alpha, n}= & \int_{-(T / 2)}^{T / 2} x(t) \tilde{\phi}_{\alpha, n}^{*}(t) d t \\
= & \int_{-\infty}^{\infty} \tilde{x}^{(t)} \tilde{\phi}_{\alpha, n}^{*}(t) d t \\
= & \int_{-\infty}^{\infty} x(t) \sqrt{\frac{\sin \alpha-j \cos \alpha}{T}} \\
& \cdot e^{j\left(\left(t^{2}+\left(n t_{0}\right)^{2}\right) / 2\right) \cot \alpha-j n t_{0} t \csc \alpha} d t
\end{aligned}
$$

where $t_{0}=2 \pi \sin \alpha / T$. An envelope function of the coefficients $C_{\alpha, n}$ is defined as

$$
E_{\alpha}(t)=\int_{-\infty}^{\infty} x(\tilde{t}) e^{j\left(\left(\tilde{t}^{2}+t^{2}\right) / 2\right) \cot \alpha-j t \tilde{t} \csc \alpha} d \tilde{t} .
$$

From the definition of FRFT, we can obtain the FRFT of the signal $x(t)$

$$
\begin{aligned}
\mathcal{X}_{\alpha}(w)= & \int_{-\infty}^{\infty} x(t) \sqrt{\frac{1-j \cot \alpha}{2 \pi}} \\
& \cdot e^{j\left(\left(t^{2}+w^{2}\right) / 2\right) \cot \alpha-j w t \csc \alpha} d t .
\end{aligned}
$$

From (A1.2)-(A1.4), we conclude that

$$
\begin{aligned}
C_{\alpha, n} & =\sqrt{\frac{\sin \alpha-j \cos \alpha}{T}} E_{\alpha}\left(n t_{0}\right) \\
& =\sqrt{\frac{2 \pi \sin \alpha}{T}} \mathcal{X}_{\alpha}\left(n t_{0}\right) .
\end{aligned}
$$

The above is the proof of (14). In the definition of FRFS, the signal $x(t)$ is written as its FRFS expansion.

$$
\begin{aligned}
x(t)= & \sum_{n=-\infty}^{\infty} \sqrt{\frac{\sin \alpha-j \cos \alpha}{T}} E_{\alpha}\left(n t_{0}\right) \tilde{\phi}_{\alpha, n}(t) \\
= & \sum_{n=-\infty}^{\infty} E_{\alpha}\left(n t_{0}\right) \sqrt{\frac{\sin \alpha-j \cos \alpha}{T}} \\
& \cdot \sqrt{\frac{\sin \alpha+j \cos \alpha}{T}} \\
& \cdot e^{-j\left(\left(t^{2}+\left(n t_{0}\right)^{2}\right) / 2\right) \cot \alpha+j n t_{0} t \csc \alpha} \\
= & \frac{1}{T} \sum_{n=-\infty}^{\infty} E_{\alpha}\left(n t_{0}\right) e^{-j\left(\left(t^{2}+\left(n t_{0}\right)^{2}\right) / 2\right) \cot \alpha+j n t_{0} t \csc \alpha} \\
= & \frac{\csc \alpha}{2 \pi} \sum_{n=-\infty}^{\infty} E_{\alpha}\left(n t_{0}\right) \\
& \cdot e^{-j\left(\left(t^{2}+\left(n t_{0}\right)^{2}\right) / 2\right) \cot \alpha+j n t_{0} t \csc \alpha} \cdot t_{0}
\end{aligned}
$$

where $t_{0}=2 \pi \sin \alpha / T . t_{0} \rightarrow 0$ as $T \rightarrow \infty$, and the right side of (A1.6) becomes an integral.

\section{APPENDIX B}

In this Appendix, we will prove (22). To begin with, we compute the integral in (22).

$$
\begin{aligned}
\int_{-\left(\pi / T_{\mathcal{S}}\right)}^{\pi / T_{\mathcal{S}}} & e^{-j\left(\nu^{2} / 2\right) \tan \alpha} \times e^{j \nu T_{\mathcal{S}}(k-n)} d \nu \\
= & \int_{-\left(\pi / T_{\mathcal{S}}\right)}^{\pi / T_{\mathcal{S}}} e^{-j(\tan \alpha / 2)\left[\nu^{2}-2 \nu T_{\mathcal{S}}(k-n) \cot \alpha\right]} d \nu \\
= & e^{j\left(T_{\mathcal{S}}^{2} / 2\right)(k-n)^{2} \cot \alpha} \int_{-\left(\pi / T_{\mathcal{S}}\right)}^{\left.\pi / T_{\mathcal{S}}\right)} \\
& \cdot e^{-j(\tan \alpha / 2)\left[\nu-T_{\mathcal{S}}(k-n) \cot \alpha\right]^{2}} d \nu
\end{aligned}
$$

Let $y=\sqrt{j(\tan \alpha / 2)}\left[\nu-T_{s}(k-n) \cot \alpha\right]$. $d y=$ $\sqrt{j(\tan \alpha / 2)} d \nu$. The above integration becomes

$$
\begin{aligned}
& \int_{-\left(\pi / T_{s}\right)}^{\pi / T_{s}} e^{-j\left(\nu^{2} / 2\right) \tan \alpha} \times e^{j \nu T_{s}(k-n)} d \nu \\
& \quad=e^{j\left(T_{s}^{2} / 2\right)(k-n)^{2} \cot \alpha} \sqrt{\frac{2 \cot \alpha}{j}} \int_{a}^{b} e^{-y^{2}} d y
\end{aligned}
$$

where

$$
\begin{aligned}
a & =\sqrt{j \frac{\tan \alpha}{2}}\left[-\frac{\pi}{T_{s}}-T_{s}(k-n) \cot \alpha\right] \\
b & =\sqrt{j \frac{\tan \alpha}{2}}\left[\frac{\pi}{T_{s}}-T_{s}(k-n) \cot \alpha\right] .
\end{aligned}
$$

Using the definition of error function, we can further compute integration as

$$
\begin{aligned}
\int_{-\left(\pi / T_{s}\right)}^{\pi / T_{s}} & e^{-j\left(\nu^{2} / 2\right) \tan \alpha} \times e^{j \nu T_{\mathcal{S}}(k-n)} d \nu \\
= & \frac{\sqrt{\pi}}{2} e^{j\left(T_{s}^{2} / 2\right)(k-n)^{2} \cot \alpha} \\
& \cdot \sqrt{\frac{2 \cot \alpha}{j}}[\operatorname{erf}(b)-\operatorname{erf}(a)] \\
= & \frac{\sqrt{\pi}}{2} e^{j\left(T_{s}^{2} / 2\right)(k-n)^{2} \cot \alpha} \\
& \cdot \sqrt{\frac{2 \cot \alpha}{j}}[\operatorname{erf}(-b)-\operatorname{erf}(-a)] \\
= & \frac{\sqrt{\pi}}{2} \sqrt{\frac{2 \cot \alpha}{j}} \Gamma(n, k, \alpha)
\end{aligned}
$$


where $\Gamma(n, k, \alpha)$ has been defined in (23). We can conclude the results of (21) and (A2.8) to obtain the following equations:

$$
\begin{aligned}
D_{\alpha}[n]= & \frac{T_{s}}{2 \pi} \sqrt{\cos \alpha+j \sin \alpha} e^{-j\left(n^{2} T_{s}^{2} / 2\right) \sin \alpha \cos \alpha} \\
& \cdot \sum_{k=-\infty}^{\infty} x[k] \int_{-\left(\pi / T_{s}\right)}^{\pi / T_{s}} \\
& \cdot e^{-j\left(\nu^{2} / 2\right) \tan \alpha} \times e^{j \nu T_{s}(k-n)} d \nu \\
= & \frac{T_{s}}{2 \pi} \sqrt{\cos \alpha+j \sin \alpha} e^{-j\left(n^{2} T_{s}^{2} / 2\right) \sin \alpha \cos \alpha} \\
& \cdot \frac{\sqrt{\pi}}{2} \sqrt{\frac{2 \cot \alpha}{j}} \sum_{k=-\infty}^{\infty} x[k] \Gamma(n, k, \alpha) \\
= & \frac{T_{s}}{2} \sqrt{\frac{1-j \cot \alpha}{2 \pi}} \sqrt{\cos \alpha} e^{-j\left(n^{2} T_{s}^{2} / 2\right) \sin \alpha \cos \alpha} \\
& \cdot \sum_{k=-\infty}^{\infty} x[k] \Gamma(n, k, \alpha) .
\end{aligned}
$$

Equation (22) has been proved.

\section{REFERENCES}

[1] A. V. Oppenheim and A. S. Willsky, Signals and Systems. Englewood Cliffs, NJ: Prentice-Hall, 1983.

[2] A. V. Oppenheim and R. W. Schafer, Discrete-Time Signal Processing. Englewood Cliffs, NJ: Prentice-Hall, 1989.

[3] L. B. Almeida, "The fractional Fourier transform and time-frequency representation," IEEE Trans. Signal Processing, vol. 42, pp. 3084-3091, Nov. 1994.

[4] S. C. Pei and M. H. Yeh, "Improved discrete fractional Fourier transform," Opt. Lett., vol. 22, pp. 1047-1049, July 15, 1997.

[5] B. Santhanam and J. H. McClellan, "The discrete rotational Fourier transform," IEEE Trans. Signal Processing, vol. 42, pp. 994-998, Apr. 1996.

[6] H. M. Ozaktas, O. Arikan, A. Kutay, and G. Bozdagi, "Digital computation of the fractional Fourier transform," IEEE Trans. Signal Processing, vol. 44, pp. 2141-2150, Sept. 1996.

[7] H. M. Ozaktas, "Fractional Fourier domains," Signal Process., vol. 46 , pp. 119-124, 1995

[8] M. A. Kutay, H. M. Ozaktas, L. Onural, and O. Arikan, "Optimal filtering in fractional Fourier domains," in Proc. IEEE Int. Conf. Acoust. Speech, Signal Processing, Detroit, MI, 1995, pp. 937-940.

[9] A. W. Lohmann, "Image rotation, Wigner rotation, and the fractiona Fourier transform," J. Opt. Soc. Amer. A, vol. 10, pp. 2181-2186, 1993.

[10] H. M. Ozaktas, B. Barshan, D. Mendlovic, and L. Onural, "Convolution, filtering, and multiplexing in fractional Fourier domains and their relation to chirp and wavelet transforms," J. Opt. Soc. Amer. A, vol 11, pp. 547-560, 1994.

[11] R. G. Dorsch, A. W. Lohmann, Y. Bitran, and D. Mendlovic, " Chirp filtering in the fractional Fourier domain," Appl. Opt., vol. 33, pp. 7599-7602, 1994.

[12] M. Abramowitz and I. A. Stegun, Handbook of Mathematical Functions, With Formula, Graphs and Mathematical Tables. New York: Dover, 1965.

\section{Novel Interpretation of the Pencil-of-Functions Approximation/Identification Method}

Pascale Bréhonnet, Riwal Morvan, Pierre Vilbé, and Léon-Claude Calvez

\begin{abstract}
Jain's pencil-of-functions method based on the linear dependence/independence of a set of functions is revisited. It is shown that no matter what model order is chosen, every estimated denominator coefficient may be regarded as the geometric mean of two values obtained in a least-squares sense.
\end{abstract}

Index Terms - Gram matrix, identification, least-squares approximation, modeling, Pencil-of-Functions, pole estimation.

\section{INTRODUCTION}

Since the pioneering work of [1], [2], generalized in [3], Jain's pencil-of-functions (POF) method of approximation/identification has received considerable attention. The resulting noniterative closedform solution gives accurate results despite not requiring any prior estimates. Pole-zero modeling of signals, such as an electromagneticscatterer response, is considered in [4] and [5] while constructing reduced-order models of a given original system, and related topics are considered in [6]-[9]. In the particular noise-free case and when the system order $n$ is correctly chosen (equal to its true value $n_{0}$ ), it has been shown [10] that the POF method minimizes a weighted version of the equation error. However, a correct choice of the system order is an essential restriction for this property to hold and, as far as the authors are aware, there is no known optimal interpretation of the POF solution when the model order $n$ is chosen less than the original system order $n_{0}$. It is the purpose of this correspondence to show that every coefficient of the characteristic equation produced by the POF method can be viewed as the geometric mean of two candidates, each candidate being the best coefficient in the sense of minimizing an equation error energy, no matter what model order $n \leqslant n_{0}$ is chosen.

\section{Assumptions, Notation, AND Problem Formulation}

Given any matrix $M$, we denote by $M^{i \Leftrightarrow j}$ the matrix obtained from $M$ by interchanging the $i$ th column and the $j$ th column while $M^{i \rightarrow j}$ is just $M$ with its $i$ th column shifted to the $j$ th position, e.g., if $M=\left[\begin{array}{lllll}c_{1} & c_{2} & c_{3} & c_{4} & c_{5}\end{array}\right]$, then $M^{2 \rightarrow 4}=\left[\begin{array}{lllll}c_{1} & c_{3} & c_{4} & c_{2} & c_{5}\end{array}\right]$ and $M^{2 \Leftrightarrow 4}=\left[\begin{array}{lllll}c_{1} & c_{4} & c_{3} & c_{2} & c_{5}\end{array}\right]$. The matrix obtained from $M$ by deleting the $i$ th row and the $j$ th column is denoted as $M_{i j}$.

To achieve an unified treatment that is valid for both continuous and discrete time signals, inner product notation is used. Given two well-behaved real-valued functions $f(t)$ and $g(t)$ of the real variable $t$ defined over some (possibly infinite) interval $\left[t_{0}, t_{K}\right]$, it is assumed that their inner product is defined, according to the nature of the problem under consideration, through the integral $\langle f, g\rangle \triangleq \int_{t_{0}}^{t_{K}} f(t) g(t) d t$ or through the discrete sum $\langle f, g\rangle \triangleq \Sigma_{k=0}^{K} f\left(t_{k}\right) g\left(t_{k}\right)$, where $t_{k}(k=0,1, \cdots, K)$ denote

Manuscript received August 4, 1998; revised April 16, 1999. The associate editor coordinating the review of this paper and approving it for publication was Prof. Jonathon A. Chambers.

The authors are with the Laboratoire d'Electronique et Systèmes de Télécommunications, Faculté des Sciences et Techniques, UMR CNRS 6616 , Université de Bretagne Occidentale (UBO), Brest, France.

Publisher Item Identifier S 1053-587X(99)07666-7. 